\title{
Solving One Dimensional Ground Water Recharge Through Porous Media Using Elzaki Transform
}

\author{
Krima B. Patel ${ }^{1}$ and Dr. Kamlesh A. Patel ${ }^{2}$
}

${ }^{1}$ Research Scholar, Mathematics Department, Shri U. P. Arts, Smt. M. G. Panchal Science \& Shri V. L. Shah Commerce college, Pilvai, India

${ }^{2}$ Associate Professor, Mathematics Department, Shri U. P. Arts, Smt. M. G. Panchal Science \& Shri V. L. Shah

Commerce college, Pilvai, India

1 Email: krimapatel223@gmail.com

²Corresponding Author; Email: kapatel.math@gmail.com

\begin{abstract}
Article Info

Volume 8, Issue 5

Page Number : 370-376

Publication Issue

September-October-2021

Article History

Accepted : 01 Oct 2021

Published : 14 Oct 2021

In this paper, we have discussed the application of Elzaki Transform for finding the solution of One-Dimensional Ground Water Recharge through porous media. In this work, we present a reliable combination of Elaki transform and Adomian Decomposition method. The proposed method introduces Adomian polynomials and the nonlinear terms can be handled by the use of this polynomials easily. The Elaki Decomposition Method is used to solve the particular problem. The purpose of this method is to extend the application of Elzaki Decomposition Method. The proposed method worked perfectly to find the One-Dimensional Ground Water Recharge through porous media problem. We obtain numerical solution and graphical representation.
\end{abstract}

Keywords : Elzaki Transform, Decomposition Method, Porous Media.

\section{INTRODUCTION}

The nonlinear partial differential equations appear in many applications of sciences and engineering such as, fluid dynamics, plasma physics, hydrodynamics, soil state physics and other disciplinaries. The ground water flow plays an important role in fluid mechanics, ground water hydrology and other fields of fluids. Such problems are also useful to measure moisture content of water in One-Dimensional Ground Water Recharge and dispersion of any fluid in porous media.
The mathematical formulation of the phenomena leads to governing equation, which is nonlinear partial differential equation. This type of equations can be solved by Elzaki Transform [1] or combined methods with Elzaki Transform [2,3,4,5]. This phenomenon has been discussed by many researches taking different viewpoints. Here are some of them, One-Dimensional Ground Water Recharge through porous media is solved by RDTM by P.S. Patel [9], Elzaki transform method is used to solve OneDimensional Ground Water Recharge through 
spreading in [10], Priti A. Tandel and P.H. Elzaki Transform is incapable to handle nonlinear Bhathawala [11] obtained solution using Crank equations easily because of the difficulties that are Nikolson method. Kunjn Shah and Twinkle Singh [12] caused by the nonlinear terms. Various ways have have studied the problem using q- Homotopy Analysis method. Verma [13] has used Laplace transform technique. A.K. Parikh, M.N. Mehta, V.H. been proposed to deal with nonlinear terms, one of these combinations is Elzaki decomposition Method, which is studied in this paper.

Pradhan [14] have obtained transcendental solution of Ground Water Recharge problem.

\section{METHODOLOGY}

\subsection{Elzaki Transform}

A New integral transform called Elzaki transform defined for functions of exponential order is proclaimed. We consider functions in the set A defined by

$$
A=\left\{f(t): \exists M, K_{1}, K_{2}>0,|f(t)|<M e^{\frac{|t|}{K_{j}}}, \text { if } t \in(-1)^{j} \times[0, \infty)\right\}
$$

Definition: If $f(t)$ is function defined for all $t \geq 0$, its Elzaki transform is the integral of $f(t)$ times $e^{-\frac{t}{v}}$ from $t=0$ to $\infty$. It is a function of $v$ and is defined as

$$
\begin{aligned}
& \qquad E[f(t)]=T(v)=v \int_{0}^{\infty} f(t) e^{-\frac{t}{v}} d t, \quad v \in\left(K_{1}, K_{2}\right) \\
& \text { Or equivalently } \quad T(v)=v^{2} \int_{0}^{\infty} f(v t) e^{-t} d t, \quad K_{1}, K_{2}>0
\end{aligned}
$$

\section{Properties of Elzaki transform}

1. $E(1)=v^{2}$

2. $E\left(t^{n}\right)=n ! v^{n+2}$

3. $E(t)=v^{3}$

4. $E^{-1}\left(v^{n+2}\right)=\frac{t^{n}}{n !}$

5. $E\left[u^{(m)}(t)\right]=\frac{1}{v^{m}} T(x, v)-\sum_{k=0}^{m-1} v^{2-m-k} u^{(k)}(0), m \geq 1$

\subsection{Elzaki Decomposition Method}

Consider a general nonlinear differential equation

$\mathrm{L} S_{w}(x, \mathrm{~T})=\mathrm{N} S_{w}(x, \mathrm{~T})+\mathrm{g}(x, \mathrm{~T})$

Where, $\mathrm{L}$ is the $1^{\text {st }}$ order linear differential operator. $\mathrm{N}$ is the nonlinear differential operator, $S_{w}$ is the dependent variable, $x$ and T are independent variables, $\mathrm{g}(x, \mathrm{~T})$ is the source term.

Apply Elzaki Transform to the equation (i)

$\mathrm{E}\left(\mathrm{L} S_{w}(x, \mathrm{~T})\right)=\mathrm{E}\left(\mathrm{N} S_{w}(x, \mathrm{~T})\right)+\mathrm{E}(\mathrm{g}(x, \mathrm{~T}))$

Using differentiation property of the Elzaki Transform, we have

$\frac{\mathrm{E}\left(S_{w}(x, \mathrm{~T})\right)}{v}-v S_{w 0}(x, \mathrm{~T})=\mathrm{E}\left(\mathrm{N} S_{w}(x, \mathrm{~T})\right)+\mathrm{E}(\mathrm{g}(x, \mathrm{~T}))$

$\mathrm{E}\left(S_{w}(x, \mathrm{~T})\right)=v^{2} S_{w 0}(x, \mathrm{~T})+v E\left(\mathrm{~N} S_{w}(x, \mathrm{~T})\right)+v \mathrm{E}(\mathrm{g}(x, \mathrm{~T}))$

Using inverse Elzaki Transform on both side of the equation (ii), we get

$S_{w}(x, \mathrm{~T})=\mathrm{G}(x, \mathrm{~T})+E^{-1}\left[v \mathrm{E}\left(\mathrm{N} S_{w}(x, \mathrm{~T})\right)\right]$

Where, $\mathrm{G}(x, \mathrm{~T})$ represents the term arising from the source term and the prescribed initial conditions.

The representation of the solution (iii) as an infinite series is given below, 


$$
S_{w}(x, \mathrm{~T})=\sum_{N=0}^{\infty} S_{w n}(x, \mathrm{~T})
$$

The non-linear term has been decomposed as:

$\mathrm{N} S_{w}(x, \mathrm{~T})=\mathrm{An}_{\mathrm{n}}\left(S_{w 0}, S_{w 1} S_{w 2}, \ldots . S_{w n}\right)$

Where $A_{n}$ are the Adomian polynomials of functions $S_{w 0}, S_{w 1} S_{w 2}, \ldots . S_{w n}$ and can be calculated by formula given as:

$$
\mathrm{A}_{\mathrm{n}}=\frac{1}{n !} \frac{\partial^{n}}{\partial \lambda^{n}}\left[\sum_{k=0}^{\infty} \lambda^{k} S_{w k}\right]_{\lambda=0} \quad \mathrm{n}=0,1,2, \ldots \ldots
$$

Substituting (iv) and (v) into (iii),

$S_{w n}(x, \mathrm{~T})=\mathrm{G}(x, \mathrm{~T})+\mathrm{E}^{-1}\left[v \mathrm{E}\left(\mathrm{A}_{\mathrm{n}}\right)\right]$

Where, $S_{w 0}(x, \mathrm{~T})=\mathrm{G}(x, \mathrm{~T})$

$$
S_{w n+1}(x, \mathrm{~T})=\mathrm{E}^{-1}\left[v \mathrm{E}\left(\mathrm{A}_{\mathrm{n}}\right)\right] \quad \mathrm{n}=0,1,2, \ldots \ldots .
$$

Where, the Elzaki Transform and the inverse Elzaki Transform are applied on (vi) respectively, the iteration $S_{w 0}, S_{w 1} S_{w 2}, \ldots . S_{w n}$ were obtained, which in turn gave the general solution as,

$S_{w}(x, \mathrm{~T})=S_{w 0}(x, \mathrm{~T})+S_{w 1}(x, \mathrm{~T})+S_{w 2}(x, \mathrm{~T})+$

\section{Problem: One Dimensional Ground Water Recharge Through Porous Media with Parabolic Permeability}

\subsection{Statement of the Problem}

In the investigated mathematical model, we consider that the groundwater recharge takes place over a large basin of such geological location that sides are limited by rigid boundaries and the bottom by a thick layer of water table. In this case, take flow is assumed vertically downloads through unsaturated porous media. It is assumed that diffusivity coefficient is equivalent to its average value over the whole range of moisture content, and the permeability of the media is continuous linear function of the problem yield a nonlinear partial differential equation for the moisture content.

\subsection{Formulation of the Problem}

We may write fundamental equation as below. The equation of continuity for unsaturated medium is given by

$$
\frac{\partial}{\partial t}\left(\rho_{s} \theta\right)=-\nabla M
$$

Where $\rho_{s}$ is bulk density of the medium, $\theta$ is the moisture content on a dry weight basis, and $M$ is the mass flux of moisture.

From Darcy's law for the motion of water in a porous media we get,

$$
V=-k \nabla \emptyset
$$

Where $\nabla \emptyset$ represent the gradient of whole moisture potential, $V$ the volume flux of moister potential, $k$ the coefficient of aqueous conductivity. Combining above two equations we obtain,

$$
\frac{\partial}{\partial t}\left(\rho_{s} \theta\right)=-\nabla(\rho k \nabla \emptyset)
$$

Where, $\rho$ is the fluid density. Since in the present case we consider that the flow takes place only in the vertical direction, equation (3) reduces to,

$$
\rho_{x} \frac{\partial \theta}{\partial t}=\frac{\partial}{\partial z}\left(\rho k \frac{\partial \varphi}{\partial z}\right)-\frac{\partial}{\partial z}(\rho k g)
$$

Where $\varphi$ is the capillary pressure potential, $g$ is the gravitational constant and $\varnothing=\varphi-g z$.

The positive direction of the $\mathrm{z}$-axis is the same as that of the gravity.

Considering $\theta$ and $\varphi$ to be conducted by single valued function, we may write (4) as,

$$
\frac{\partial \theta}{\partial t}=\frac{\partial}{\partial z}\left(D \frac{\partial \theta}{\partial z}\right)-\frac{\rho}{\rho_{s}} g \frac{\partial k}{\partial z}
$$

Where $D=\frac{\rho}{\rho_{s}} k \frac{\partial \varphi}{\partial \theta}$ and is called diffusivity coefficient.

Replacing D by its average value $D_{a}$ and assuming $k=k_{0} \theta, k_{0}=0.232$, we have

$$
\frac{\partial \theta}{\partial t}=D_{a} \frac{\partial^{2} \theta}{\partial z^{2}}-\frac{\rho}{\rho_{s}} k_{0} \frac{\partial \theta}{\partial z}
$$


Considering water table to be situated at a depth $\mathrm{L}$, and putting,

$\frac{z}{L}=\xi, \frac{t D_{a}}{L^{2}}=T, \beta_{0}=\frac{\rho}{\rho_{s}} k_{0} \frac{\partial \theta}{\partial z}$

One-dimensional Ground water recharge through porous media with linear permeability is,

$$
\frac{\partial \theta}{\partial T}=\frac{\partial^{2} \theta}{\partial \xi^{2}}-\beta_{0} \frac{\partial \theta}{\partial \xi}
$$

Where $\xi=$ penetration depth (dimensionless)

$$
\begin{gathered}
T=\text { time (dimensionless) } \\
\beta_{0}=\text { flow parameter }\left(\mathrm{cm}^{2}\right)
\end{gathered}
$$

Set of appropriate boundary conditions are $\theta(0, T)=\theta_{0}, \theta(1, T)=1, \theta(\xi, 0)=0$

But here we assumed, the permeability of the moisture content to have a parabolic distribution, i.e. $k=k_{i}+k_{0} \theta^{2}\left(k_{0}=0.232\right)$ where $k_{i}$ and $k_{0}$ are constants. Then equation (5) becomes,

$$
\frac{\partial \theta}{\partial t}=D_{a} \frac{\partial^{2} \theta}{\partial z^{2}}-\frac{\rho}{\rho_{s}} k_{0} \frac{\partial \theta}{\partial z}
$$

Considering the water table to be situated at a depth $\mathrm{L}$, and put,

$\frac{z}{L}=\xi, \frac{t D_{a}}{L^{2}}=T, \beta_{0}=\frac{\rho}{\rho_{s}} \frac{k_{0}}{D_{a}}$

We may write the problem as below from the boundary value

$$
\frac{\partial \theta}{\partial T}=\frac{\partial^{2} \theta}{\partial \xi^{2}}-\beta_{0} \theta \frac{\partial \theta}{\partial \xi}
$$

It may be mentioned for definiteness that a set of appropriate boundary conditions are

$\theta(0, T)=\theta_{0}, \theta(1, T)=1, \theta(\xi, 0)=0$

Where the moisture content throughout the region is zero initially, at the layer $\mathrm{z}=0$ it is $\theta_{0}$ and at the water table $\mathrm{z}=\mathrm{L}$ it is assumed to remain $100 \%$ throughout the process of investigation. It may be remarked that the effect of capillary action at the stationary ground water level, being small is neglected.

We can write equation (7) as $\theta_{T}=\theta_{\xi \xi}-\beta_{0} \theta \theta_{\xi}$

This problem is solved by Elzaki Decomposition Method and numerical solution is obtained. The graph indicates the moisture content corresponding to various time periods.

\subsection{Solution using Elzaki Decomposition Method}

$$
\theta_{T}=\theta_{\xi \xi}-\beta_{0} \theta \theta_{\xi}
$$

Taking initial condition $\theta(\xi, 0)=\theta_{0}=f(\xi)=\frac{e^{\xi}-1}{e-1}$

This equation is nonlinear differential equation, which is solved by Elzaki Decomposition Method.

Apply Elzaki transform on (1)

$$
E(\theta)=v^{2} \theta_{0}+v E\left[\left[\theta_{\xi \xi}-\beta_{0} \theta \theta_{\xi}\right]\right]
$$

Now, applying inverse Elzaki transform,

$$
\begin{aligned}
& \theta=\theta_{0}+E^{-1}\left[v E\left(\theta_{\xi \xi}-\beta_{0} \theta \theta_{\xi}\right)\right] \\
& \therefore \theta=\theta_{0}+E^{-1}\left[v E\left(\varnothing_{1}-\beta_{0} \varnothing_{2}\right)\right]
\end{aligned}
$$

Where $\emptyset_{1}=\sum_{\mathrm{n}=0}^{\infty} \mathrm{A}_{\mathrm{n}}$ and $\emptyset_{2}=\sum_{\mathrm{n}=0}^{\infty} \mathrm{B}_{\mathrm{n}}$$$
\therefore \theta=\theta_{0}+E^{-1}\left[v E\left(\sum_{\mathrm{n}=0}^{\infty}\left(\mathrm{A}_{\mathrm{n}}-\beta_{0} \mathrm{~B}_{\mathrm{n}}\right)\right)\right]
$$

The Adomian polynomials $A_{n}$ and $B_{n}$ are calculated as

$$
\mathrm{A}_{\mathrm{n}}=\frac{1}{n !} \frac{\partial^{n}}{\partial \lambda^{n}}\left[\emptyset_{1}\left(\sum_{\mathrm{k}=0}^{\infty} \lambda^{\mathrm{k}} \theta_{k}\right)\right]_{\lambda=0} \quad \mathrm{n}=0,1,2, \ldots \ldots
$$




$$
\mathrm{B}_{\mathrm{n}}=\frac{1}{n !} \frac{\partial^{n}}{\partial \lambda^{n}}\left[\emptyset_{2}\left(\sum_{\mathrm{k}=0}^{\infty} \lambda^{\mathrm{k}} \theta_{k}\right)\right]_{\lambda=0} \quad \mathrm{n}=0,1,2, \ldots \ldots
$$

The first three components of these polynomials are

$$
\begin{aligned}
\mathrm{A}_{0} & =\left(\theta_{0}\right)_{\xi \xi} \\
\mathrm{A}_{1} & =\left(\theta_{1}\right)_{\xi \xi} \\
\mathrm{A}_{2} & =\left(\theta_{2}\right)_{\xi \xi} \\
\mathrm{B}_{0} & =\theta_{0}\left(\theta_{0}\right)_{\xi} \\
\mathrm{B}_{1} & =\theta_{0}\left(\theta_{1}\right)_{\xi}+\theta_{1}\left(\theta_{0}\right)_{\xi} \\
\mathrm{B}_{2} & =\theta_{0}\left(\theta_{2}\right)_{\xi}+\theta_{1}\left(\theta_{1}\right)_{\xi}+\theta_{2}\left(\theta_{0}\right)_{\xi}
\end{aligned}
$$

Other polynomials can be generated in like this manner, substituting this decomposition series in (3) and taking $\beta_{0}=1$

$$
\begin{aligned}
& \theta_{0}=\theta_{0}=\frac{e^{\xi}-1}{e-1} \\
& \theta_{1}=E^{-1}\left[v E\left(\mathrm{~A}_{0}-\mathrm{B}_{0}\right)\right] \\
& =E^{-1}\left[v E\left(\left(\theta_{0}\right)_{\xi \xi}-\theta_{0}\left(\theta_{0}\right)_{\xi}\right)\right] \\
& =\frac{e^{\xi+1}-e^{2 \xi}}{(e-1)^{2}} \mathrm{~T}^{1} \\
& \theta_{2}=E^{-1}\left[v E\left(\mathrm{~A}_{1}-\mathrm{B}_{1}\right)\right] \\
& =E^{-1}\left[v E\left(\left(\theta_{1}\right)_{\xi \xi}-\theta_{0}\left(\theta_{1}\right)_{\xi}-\theta_{1}\left(\theta_{0}\right)_{\xi}\right)\right] \\
& =\frac{3 e^{3 \xi}-6 e^{2 \xi+1}+2 e^{2 \xi}+e^{\xi+2}}{2(e-1)^{3}} \mathrm{~T}^{2} \\
& \theta_{3}=E^{-1}\left[v E\left(\mathrm{~A}_{2}-\mathrm{B}_{2}\right)\right] \\
& \quad=E^{-1}\left[v E\left(\left(\theta_{2}\right)_{\xi \xi}-\theta_{0}\left(\theta_{2}\right)_{\xi}-\theta_{1}\left(\theta_{1}\right)_{\xi}-\theta_{2}\left(\theta_{0}\right)_{\xi}\right)\right] \\
& =\frac{-14 e^{4 \xi}+48 e^{3 \xi+1}-24 e^{3 \xi}-27 e^{2(\xi+1)}+20 e^{2 \xi+1}-4 e^{2 \xi}+e^{\xi+3}}{6(e-1)^{4}} \mathrm{~T}^{3}
\end{aligned}
$$

Thus, the final solution is given as

$$
\begin{aligned}
\theta(\xi, T)= & \sum_{\mathrm{n}=0}^{\infty} \theta_{n}(\xi, T) \\
= & \frac{e^{\xi}-1}{e-1}+\frac{e^{\xi+1}-e^{2 \xi}}{(e-1)^{2}} \mathrm{~T}^{1}+\frac{3 e^{3 \xi}-6 e^{2 \xi+1}+2 e^{2 \xi}+e^{\xi+2}}{2(e-1)^{3}} \mathrm{~T}^{2}+ \\
& \frac{-14 e^{4 \xi}+48 e^{3 \xi+1}-24 e^{3 \xi}-27 e^{2(\xi+1)}+20 e^{2 \xi+1}-4 e^{2 \xi}+e^{\xi+3}}{6(e-1)^{4}} \mathrm{~T}^{3}+\cdots
\end{aligned}
$$

\section{NUMERICAL SOLUTION}

The following table shows the approximate moisture content of liquid for different values of depth at different time using Elzaki decomposition method.

\begin{tabular}{|c|c|c|c|c|c|c|c|}
\hline$\xi$ & $\mathrm{T}=0$ & $\mathrm{~T}=0.1$ & $\mathrm{~T}=0.2$ & $\mathrm{~T}=0.3$ & $\mathrm{~T}=0.4$ & $\mathrm{~T}=0.5$ & $\mathrm{~T}=0.6$ \\
\hline 0 & 0 & 0 & 0 & 0 & 0 & 0 & 0 \\
\hline 0.1 & 0.06121 & 0.12159 & 0.18197 & 0.24235 & 0.30273 & 0.36312 & 0.4235 \\
\hline 0.2 & 0.12885 & 0.19077 & 0.2527 & 0.31462 & 0.37655 & 0.43847 & 0.50039 \\
\hline 0.3 & 0.20361 & 0.26617 & 0.32874 & 0.3913 & 0.45386 & 0.51643 & 0.57899 \\
\hline 0.4 & 0.28623 & 0.3482 & 0.41017 & 0.47214 & 0.53411 & 0.59608 & 0.65805 \\
\hline 0.5 & 0.37754 & 0.43727 & 0.49699 & 0.55672 & 0.61644 & 0.67617 & 0.7359 \\
\hline 0.6 & 0.47845 & 0.53376 & 0.58907 & 0.64437 & 0.69968 & 0.75499 & 0.81029 \\
\hline 0.7 & 0.58998 & 0.63803 & 0.68609 & 0.73414 & 0.78219 & 0.83024 & 0.8783 \\
\hline 0.8 & 0.71324 & 0.75038 & 0.78752 & 0.82466 & 0.8618 & 0.89895 & 0.93609 \\
\hline 0.9 & 0.84946 & 0.871 & 0.89255 & 0.9141 & 0.93565 & 0.9572 & 0.97875 \\
\hline 1 & 1 & 1 & 1 & 1 & 1 & 1 & 1 \\
\hline
\end{tabular}




\section{MOISTURE CONTENT AT DIFFERENT TIME}

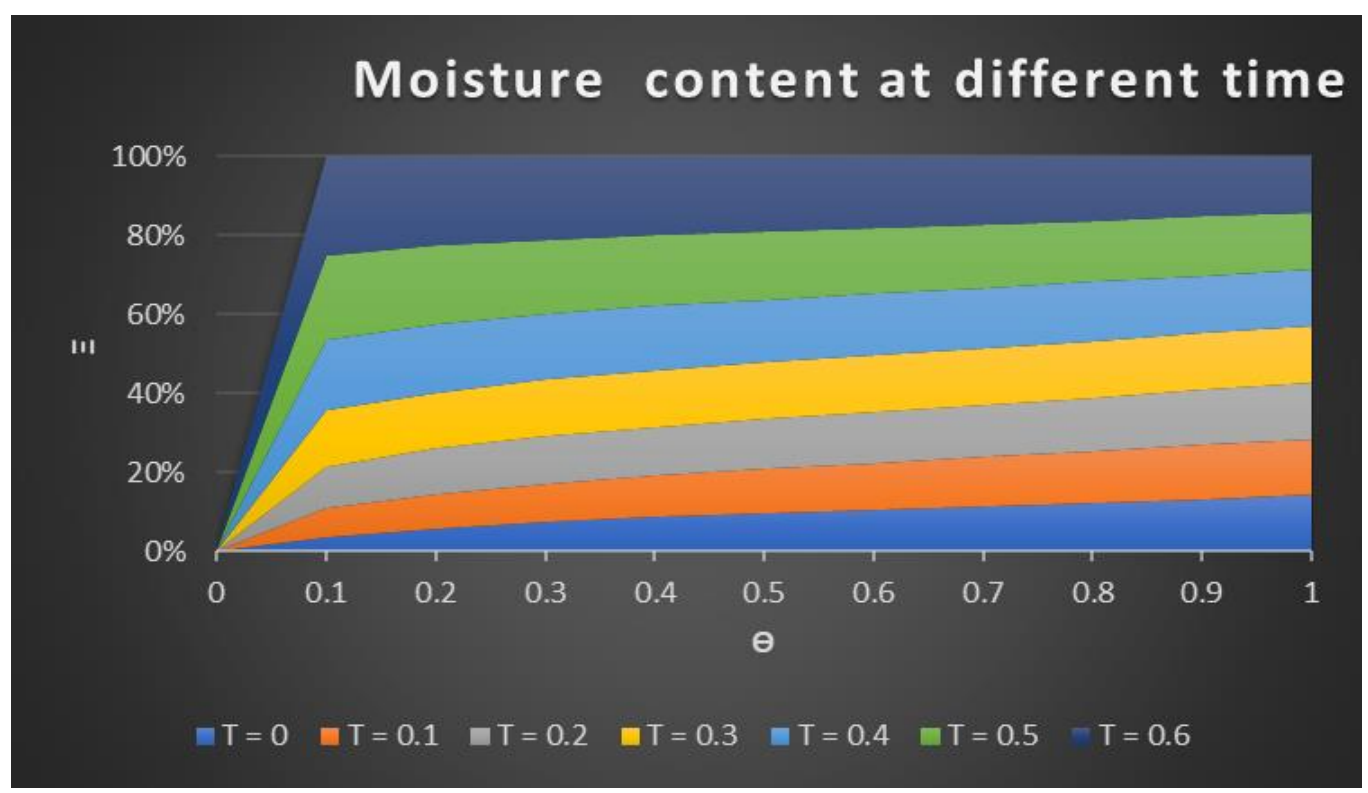

\section{Conclusion}

In the graph $\mathrm{X}$-axis represents moisture content and $\mathrm{Y}$-axis represents depth. It's shows that as time $\mathrm{T}$ increases, the moisture content also increases at each point of the basin and after some time, it become constant.

\section{VII.Acknowledgements}

The support from the SHODH, Government of Gujarat for giving financial support is highly acknowledged. The author is grateful to P. S. Patel for constructive criticism that significantly contributed in improving the quality of the article.

\section{REFERENCES}

[1]. Elzaki Tarig, Salih M. Elzaki, On the New Integral Transform "Elzaki Transform" Fundamental Properties Investigations and Applications, Global Journal of Mathematical Sciences: Theory and Practical, 4 (1) (2012) 113.
[2]. Tarig M. Elzaki, Eman M. A. Hilal, Homotopy Perturbation and Elzaki Transform for Solving Nonlinear Partial Differential Equations, Mathematical Theory and Modeling, 2 (3) (2012) 33-42.

[3]. Shraddha S. Chavan, Mihir M. Panchal, Solution of Porous Medium Equation Arising in Fluid Flow through Porous Media by Homotopy Perturbation Method Using Elzaki Transform, International Journal of Futuristic Trends in engineerimg and Technology, 1 (10) (2014) 3235.

[4]. Mousumi Datta, Umme Habiba, Md. Babul Hossain, Elzaki Substitution Method for Solving Nonlinear Partial Differential Equations with Mixed Partial Derivatives Using Adomain Polynomial, International Journal of Partial Differential Equations And applications, 8 (1) (2020) 6-12.

[5]. Yunqi Xu, Dianchen Lu, Study on Approximate Solution of Fractional Order Biological Population Model, Archives of Current Research International, 15 (4) (2018) 1-11. 
[6]. D. B. Dhaigude, Gunvant A. Birajdar, V. R. Nikam, ADOMIAN DECOMPOSITION METHOD FOR FRACTIONAL BENJAMINBONA-MAHONY-BURGER'S EQUATIONS, Int. J. of Appl. Math., 8 (12) (2012) 42-51.

[7]. Shams A. Ahmed, Tarig M. Elzaki, The Solution of Nonlinear Volterra Integro-Differential Equations of Second Kind by Combine Sumudu Transform and Adomain Decomposition Method, International Journal of Advanced and Innovative Research, 2 (12) (2013) 90-93.

[8]. M. A. Helal, M. S. Mehanna, The tanh method and Adomian decomposition method for solving the foam drainage equation, Applied Mathematics and Computation, 190 (2007) 599609.

[9]. P.S. Patel, Application of Reduced differential transform method to fluid flow through porous medium, PhD Thesis, PAHER University Udaipur, Rajasthan, India, (2017).

[10]. Prem Kiran G. Bhadane, V. H. Pradhan, Satish V. Desale, Elzaki Transform Solution of One Dimensional Ground Water Recharge through Spreading, Int. Journal of Engineering Research and Applications, 3 (6) (2013) 1607-1610.

[11]. Priti A. Tandel, P. H. Bhathawala, OneDimensional ground water recharge through porous media, International Journal of Advances in Applied Mathematics and Mechanics, 1 (1) (2013) 61-67.

[12]. Kunjan Shah, Twinkle Singh, Solution of Burger's Equation in a One-Dimensional Groundwater Recharge by Spreading Using qHomotopy Analysis Method, EUROPEAN JOURNAL OF PURE AND APPLIED MATHEMATICS, 9 (1) (2016) 114-124.

[13]. A. P. Verma, The Laplace transform solution of one-dimensional groundwater recharge by spreading, Annali Di Geofision, 22 (1) (1969) 25-31.

[14]. A. K. Parikh, M. N. Mehta, V. H. Pradhan, TRANCEDENTAL SOLUTION OF FOKKER-
PLANCK EQUATION OF VERTICAL GROUND WATER RECHARGE IN UNSATURATED HOMOGENIOUS POROUS MEDIA, International Journal of Engineering Research and Application (IJERA), 1 (4) 19041911.

\section{Cite this article as :}

Krima B. Patel, Dr. Kamlesh A. Patel, "Solving One Dimensional Ground Water Recharge Through Porous Media Using Elzaki Transform", International Journal of Scientific Research in Science and Technology (IJSRST), Online ISSN : 2395-602X, Print ISSN : 2395-6011, Volume 8 Issue 5, pp. 370-376, September-October 2021. Available at doi : https://doi.org/10.32628/IJSRST218557 Journal URL : https://ijsrst.com/IJSRST218557 